\title{
«Herméneutique et Tradition» au Colloque de Rome
} Rudolf Boehm

\section{Citer ce document / Cite this document :}

Boehm Rudolf. «Herméneutique et Tradition» au Colloque de Rome. In: Revue Philosophique de Louvain. Troisième série, tome $61, \mathrm{n}^{\circ} 71,1963$. pp. 467-468;

doi : https://doi.org/10.3406/phlou.1963.5219

https://www.persee.fr/doc/phlou_0035-3841_1963_num_61_71_5219

Fichier pdf généré le 25/04/2018 


\section{«Herméneutique et Tradition» au Colloque de Rome}

Toute tradition (au sens actif du mot) - l'accord semble assez général à ce propos - est signi-fication (au sens actif du mot également). Toute interprétation d'une signification est-elle autre chose qu'explicitation de celle-ci, et toute herméneutique autre chose qu'explicitation de l'interprétation elle-même ? En sorte que toute interprétation impliquerait son herméneutique, toute signification son interprétation et donc, déjà, une herméneutique ? Bref, toute tradition et, en ce sens et dans cette mesure, toute histoire, ne seraient-elles qu'explicitation de l'implicite, ou bien, autrement dit, " évolution " ? En fait, la conception évolutionniste de l'histoire $s^{\prime}$ exprime aujourd'hui en termes d'herméneutique. Elle conserve du reste l'avantage considérable d'unir en elle et la certitude d'une substance de l'histoire acquise une fois pour toutes et la perspective diun avenir riche de promesses.

Par contre, il est manifeste aussi, - et par le fait même que des liens étroits relient entre elles tradition, signification, interprétation et herméneutique - , que toute entreprise d'interprétation naît du besoin de rechercher une signification qui ne nous est pas manifestée par la tradition elle-même, et que l'effort d'une herméneutique répond à la nécessité de fonder un " art " de l'interprétation lorsque celle-ci ne se trouve plus " naturellement " guidée par ce qu'une tradition lui signifie. Sans doute ne peut-on accuser l'interprétation et l'herméneutique elles-mêmes de rompre avec les traditions et de remettre en doute les significations traditionnelles, mais une rupture dans l'histoire semble être indiquée, chaque fois, par le fait de recherches d'interprétation et d'herméneutique.

Ce fut la confrontation de ces deux ordres d'idées qui anima, nous semble-t-il, les débats du Colloque sur Ermeneutica e Tradizione qui s'est tenu du 10 au 16 janvier 1963 à Rome, sous les 
auspices de l'lstituto di Studi Filosofici et du Centro Internazionale di Studi Umanistici, sous la présidence de M. Enrico Castelli, et dont les Actes ont été publiés en mai dernier ${ }^{\text {(1) }}$. Y participaient une trentaine de philosophes et de théologiens, catholiques et protestants, de plusieurs pays. Le volume des $A$ ctes reproduit le texte des conférences faites (dans l'ordre) par MM. Castelli, Ricœur, De Waelhens, Boehm, Gouhier, Ott, Kerényi, le P. de Lubac, M. Grant, les PP. Fessard, Marlé, Theunis et Lotz, MM. Ulrich et Lazzarini, M. l'abbé Panikkar, le P. Fagone et M. Klein, ainsi que des contributions de MM. Mancini et Bartsch, des PP. Daniélou et Breton et de M. Dorfles qui n'ont pas pu être présentées au Colloque même, et enfin de très larges extraits des discussions ${ }^{(2)}$.

Citons les trois premières phrases par lesquelles $M$. Castelli commença son introduction au Colloque, rappelant d'abord par quel biais celui-ci se rattachait aux Colloques précédemment tenus sur les thèmes de la "démythisation ": "La démythisation est toujours le résultat d'une herméneutique. Une herméneutique naît de l'état d'insatisfaction. L'insatisfaction de l'insatisfaction est un non-sens $)$.

\section{Rudolf BOEHM.}

Louvain.

(1) Ermeneatica e Tradizione. Atti del Convegno indetto dal Centro Internazionale di Studi Umanistici e dall'Istituto di Studi Filosofici, Roma - 10-16 Gennaio 1963, a cura di Enrico Castellu, un vol. $24 \times 17$ de XVI-450 pp., Roma, Istituto di Studi Filosofici, 1963.

(2) Pour les thèmes des exposés, voir le numéro de février de cette Revue, pp. 167-168. 